\title{
Event-Triggered Observers and Observer-Based Controllers for a Class of Nonlinear Systems
}

\author{
L. Etienne, S. Di Gennaro, and J.-P. Barbot
}

\begin{abstract}
In this paper, we investigate the stabilization of a nonlinear plant subject to network constraints, under the assumption of partial knowledge of the plant state. The event triggered paradigm is used for the observation and the control of the system. Necessary conditions, making use of the ISS property, are given to guarantee the existence of a triggering mechanism, leading to asymptotic convergence of the observer and system states. The proposed triggering mechanism is illustrated in the stabilization of a robot with a flexible link robot.
\end{abstract}

\section{INTRODUCTION}

The use of the digital technology is pervasive in modern control systems, where the control task consists of the sampling of the plant outputs, the computation, and the implementation of the actuator signals. The classic way is to sample in a periodic fashion, thus allowing the closedloop system to be analysed on the basis of sampled-data systems, see [2]. Recent years have seen the development of a different paradigm where, instead of being sampled periodically (i.e. with a time-triggered policy), the system is triggered when the stability property is lost (i.e with an event-triggered policy). A good number of works deal with this subject, see [3], [12], [14], [9], [5], and [6] for an introduction to this topic. The problem is to design an eventtriggered mechanism to ensure the closed-loop stability. This problem was solved, for both the linear and the nonlinear case, when the full state is available [12], [14]. When the state is not available, the problem was addressed in [8], [4] for linear systems. In [13] the results were extended to linear event-triggered network control systems. In the nonlinear setting, to the best of the authors' knowledge, no result is still available when the whole state is not available for feedback.

The main objective of this paper is to address the problem of the event-triggered output-based feedback for nonlinear systems, giving sufficient conditions for the dynamic feedback control of nonlinear plants subject to network constraints, using an event-triggered strategy.

The paper is organized as follows. In Section II we recall the event-triggered control, and we introduce the class of systems considered. In Section III we give sufficient conditions on the observer and on the observation error in terms of input-to-state stability, along with relevant event-triggering

Lucien Etienne and S. Di Gennaro are with the Department of Information Engineering, Computer Science and Mathematics, and with the Center of Excellence DEWS, University of L'Aquila, Via Vetoio, Loc. Coppito, 67100 L'Aquila, Italy. E.mail: \{lucien.etienne, stefano.digennaro\}eunivaq.it.

Jean-Pierre Barbot is with the Ecole Nationale Superieure d'Electronique et de Ses Applications - ENSEA, 6 Avenue du Ponçeau, 95014 Cergy Pontoise Cedex E.mail: barbot@ensea.fr. mechanisms, in order to ensure asymptotic convergence to the origin. In Section IV we consider some type of systems fitting into the class of systems considered in Section III. In Section V an example is given. Finally, in Section VI we give some concluding remarks.

Notation: In the following, $|\cdot|$ denotes the norm $\|\cdot\|_{1}$, and $\|\cdot\|$ is the euclidean norm. Moreover, $|\cdot|_{\infty}$ is the component with the biggest absolute value. Furthermore, $\alpha(\cdot) \in \mathcal{K}$ if it a strictly increasing function from $[0, a) \rightarrow[0, \infty)$, while $\alpha$ is a class $\mathcal{K}_{\infty}$ function if it strictly increasing function from $[0, \infty) \rightarrow[0, \infty)$ and $\lim _{r \rightarrow \infty} \alpha(r)=\infty$. Finally, $\beta(r, s) \in$ $\mathcal{K} \mathcal{L}$ if $\beta(\cdot, s) \in \mathcal{K}$ for all $s$ and $\lim _{s \rightarrow \infty} \beta(r, s)=0$ for all $r$. When a function $f$ is Lipschitz, we denote $L_{f}$ its Lipschitz constant.

\section{PRoblem FORMULATION AND DEFINITIONS}

\section{A. Problem Statement and Event Triggering Policies}

We will first recall some known facts and terminologies about event triggered systems. Consider the system

$$
\begin{aligned}
& \dot{x}(t)=f_{s}(x(t), u(t)) \\
& y(t)=h(x(t))
\end{aligned}
$$

where $x \in \mathbb{R}^{n}$ is the state, $u \in \mathbb{R}^{m}$ is the control, $y \in \mathbb{R}^{p}$ is the output. The time instant $t$ is dropped if there are no ambiguities. The functions $f$ and $h$ are assumed sufficiently smooth. We also assume the existence of a continuous state based controller which renders the origin asymptotically stable.

The control scheme is shown in Fig. 1. Due to the communication constraints, there is no continuous communication either between sensors and observer, or between observer and actuators. The inputs and the outputs are partitioned into actuator/sensor nodes $u=\left(u_{1}^{T}, \cdots, u_{q}^{T}\right)^{T}, y=$ $\left(y_{1}^{T}, \cdots, y_{r}^{T}\right)^{T}=\left(h_{1}^{T}(x), \cdots, h_{r}^{T}(x)\right)^{T}$, with $u_{1}, \cdots, u_{q}$, $y_{1}, \cdots, y_{r}$, not necessarily scalars.

The value $y_{i}\left(t_{k_{i}}\right)=h_{i}\left(x\left(t_{k_{i}}\right)\right), i=1, \cdots, r$, is the last sampled value at the $i^{t h}$ sensor node, available for the controller to implement the control, while the value $u_{i}\left(t_{j_{i}}\right)$, $i=1, \cdots, q$, is applied to the system at the $i^{t h}$ actuator node, through a classic zero-order holder $H_{0}$. It is worth noting that this means that the different outputs $\left\{y_{i}\right\}_{i=1, \cdots, r}$ and the different inputs $\left\{u_{i}\right\}_{i=1, \cdots, q}$ are not sampled synchronously. For this reason, at time $t$ the latest output available is

$$
\bar{y}(t)=\left(y_{1}^{T}\left(t_{k_{1}}\right), y_{2}^{T}\left(t_{k_{2}}\right) \cdots, y_{p}^{T}\left(t_{k_{p}}\right)\right)^{T}
$$


while the control is

$$
\bar{u}(t)=\left(u_{1}^{T}\left(t_{j_{1}}\right), u_{2}^{T}\left(t_{j_{2}}\right) \cdots, u_{q}^{T}\left(t_{j_{q}}\right)\right)^{T} .
$$

Denoting by $e_{u}=u-\bar{u}$ and $e_{y}=y-\bar{y}$ the difference vectors between the continuous and sampled values, one considers the vector $E=\left(e_{u}^{T}, e_{y}^{T}\right)^{T}$ of the error due to the sampling.

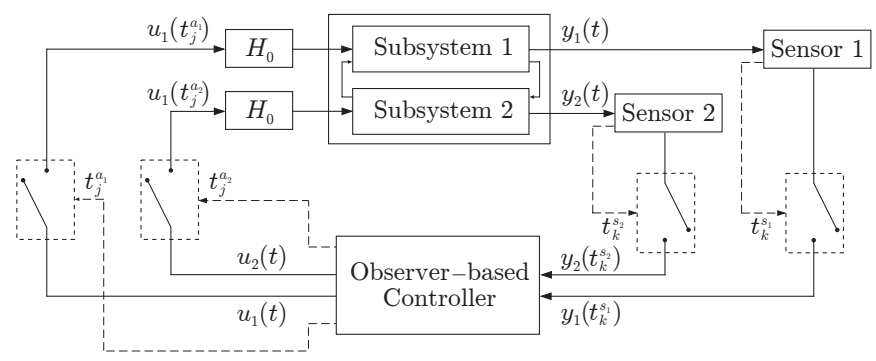

Fig. 1. Control scheme with sampled output and zero order holder

Let us consider first a simple case in which the state $x$ is available for measurement, and let us assume that there exists a state-feedback

$$
u=\gamma(x)
$$

rendering system (1) asymptotically stable at the origin. The partitioned input vector is

$$
u=\left(\gamma_{1}^{T}(x), \cdots, \gamma_{q}^{T}(x)\right)^{T} .
$$

When the controller is implemented making use of the sampled values, one considers the last communication time $t_{j}$ between controller and plant, and the control value

$$
\bar{u}=\bar{\gamma}(x)=\left(\gamma_{1}^{T}\left(x\left(t_{j_{1}}\right), \cdots, \gamma_{q}^{T}\left(x\left(t_{j_{q}}\right)\right)\right)^{T} .\right.
$$

Using a classic periodic sampling, the next sampling time is $t_{k+1}=t_{k}+\delta$, where $\delta>0$, so that $t_{k+1}-t_{k}=\delta>0$ or, that is the same

$$
t_{k+1}=\min _{t}\left\{t \mid t>t_{k}+\delta\right\} .
$$

The event triggered paradigm replaces this condition with a condition on the state values $x(t), x\left(t_{k}\right)$. A simple condition of this kind is, for instance, the epsilon crossing policy, which is of the form

$$
t_{k+1}=\min _{t}\left\{t \geq t_{k}|| x(t)-x\left(t_{k}\right) \mid>\varepsilon\right\}
$$

viz. $x(t)$ is sampled when $\left|x(t)-x\left(t_{k}\right)\right|$ is greater than a certain threshold value $\varepsilon \in \mathbb{R}$. When this condition is verified, an event is triggered, which determines the sampling time $t_{k+1}$. The difference $\delta_{k}=t_{k+1}-t_{k}$ is usually called the inter-event time. To avoid Zeno behaviors [7], it is important that the chosen sampling policy ensures that $\delta_{k}>0$ for all $k \in \mathbb{N}$, possibly under additional conditions.

Further strategies can also be used to determine the next sampling time. For instance, the state dependent triggering condition

$$
t_{k+1}=\min _{t}\left\{t \geq t_{k}|| x(t)-x\left(t_{k}\right)|>\sigma| x \mid+\varepsilon\right\}
$$

with $\varepsilon, \sigma \in \mathbb{R}^{+}$, or a mixed triggering policy

$$
t_{k+1}=\min _{t}\left\{t \geq t_{k}+\delta_{\min },|| x(t)-x\left(t_{k}\right) \mid>\varepsilon\right\}
$$

with $\varepsilon, \delta_{\min } \in \mathbb{R}^{+}$. Furthermore, (1) can be stabilized asymptotically with the state triggering condition

$$
t_{k+1}=\min _{t}\left\{t \geq t_{k},|| x(t)-x\left(t_{k}\right)|>\sigma| x(t) \mid\right\}
$$

under the sole assumption that the closed loop nonlinear system is input-to-state stable with respect to the quantity $\left|x(t)-x\left(t_{k}\right)\right|[12]$.

When the state $x$ of (1) is not measurable, these triggering policies cannot be implemented. In the following, we will introduce the triggering policy that will be used in this case, taking into account the constraints on the communication of output and input. An obvious assumption is that it is possible to design an observer that converges asymptotically to $x$, of the form

$$
\dot{\hat{x}}=f_{o}(\hat{x}, y, u) .
$$

where $f_{o}: \mathbb{R}^{n} \times \mathbb{R}^{p} \times \mathbb{R}^{m} \rightarrow \mathbb{R}^{n}$ is not smooth, in general. In view of an implementation via a triggering policy, and since the observer has not $y(t)$ available, one can use the vector $\bar{y}$, so considering the observer

$$
\dot{\hat{x}}=f_{o}(\hat{x}, u, \bar{y}) .
$$

A feedback controller based on $\hat{x}$ given by (3) will be used in the following to stabilize the system (1) in the origin. The input applied to the system, due to the communication channel, is $\bar{u}=\bar{\gamma}(\hat{x})$, so obtaining the controlled dynamics

$$
\dot{x}=f_{s}(x, \bar{\gamma}(\hat{x})) \text {. }
$$

Eventually, one gets the following closed-loop system

$$
\begin{aligned}
& \dot{x}=f_{s}(x, \bar{\gamma}(\hat{x})) \\
& \dot{\hat{x}}=f_{o}(\hat{x}, \bar{\gamma}(\hat{x}), \bar{y}) .
\end{aligned}
$$

The observation error is $z=x-\hat{x}$. We assume that the observation error dynamics can be written is the form

$$
\dot{z}=f_{s}(x, \bar{\gamma}(\hat{x}))-f_{o}(\hat{x}, \bar{\gamma}(\hat{x}), \bar{y})=g\left(z, \theta_{1}\left(e_{u}\right), \theta_{2}\left(e_{y}\right), \hat{x}\right)
$$

where $\theta_{1}, \theta_{2}$ give the dependence on the input and the output errors $e_{u}, e_{y}$, due to the sampling.

\section{B. Definitions}

Definition 1 (Input-to-state stability-ISS): System (1) is said to be locally ISS if there exist a $\mathcal{K} \mathcal{L}$ function $\beta$, a $\mathcal{K}$ function $\alpha$, and some constants $k_{1}, k_{2}>0$ such that

$$
|x(t)| \leq \beta\left(\left|x_{0}\right|, t\right)+\alpha(|u|), \quad \forall t \geq 0
$$

for all $x_{0} \in D, u \in D_{u}$ satisfying $\left|x_{0}\right|<k_{1},|u|<k_{2}$. System (1) is said (globally) input-to-state stable if $D=\mathbb{R}^{n}$, $D_{u}=\mathbb{R}^{m}$, and the above inequalities are satisfied for any initial state and any bounded input.

Definition 2 (ISS Lyapunov function):

A continuous function $V: D \rightarrow R$ is an ISS Lyapunov 
function on $D$ for system (1) if there exist class $\mathcal{K}$ functions $\alpha_{1}, \alpha_{2}, \alpha_{3}, \beta$ such that the following two conditions are satisfied

$$
\begin{array}{cl}
\alpha_{1}(|x|) \leq V(x(t)) \leq \alpha_{2}(|x|) & \forall x \in D, t \geq 0 \\
\frac{\partial V(x)}{\partial x} f(x, u) \leq-\alpha_{3}(|x|)+\beta(|u|) & \forall x \in D, u \in D_{u} .
\end{array}
$$

Moreover, $V$ is an global ISS Lyapunov function if $D=$ $R^{n}, D_{u}=R^{m}$, and $\alpha_{1}, \alpha_{2}, \alpha_{3}, \beta, \in \mathcal{K}_{\infty}$.

\section{MAIN RESULT}

A. Hypothesis on the Dynamics of the State Observer and of the Observation Error

Since the observer state is available, in the following we consider the observer dynamics, so allowing imposing on $\hat{x}$ a triggering condition, along with the observation error dynamics

$$
\begin{aligned}
\dot{\hat{x}} & =f_{o}(\hat{x}, \bar{\gamma}(\hat{x}), \bar{h}(\hat{x}+z)) \\
\dot{z} & =g\left(z, \theta_{1}\left(e_{u}\right), \theta_{2}\left(e_{y}\right), \hat{x}\right)
\end{aligned}
$$

where $y=h(\hat{x}+z)$ and $\bar{y}=\bar{h}(\hat{x}+z)$, or equivalently

$$
\dot{X}=G(X, E)
$$

where $X=\left(\hat{x}^{T}, z^{T}\right)^{T}$ is an extended state vector, and $G=\left(f_{o}^{T}, g^{T}\right)^{T}$. In the following we consider the following assumptions.

$\left(A_{1}\right)$ There exists an ISS Lyapunov function for 4a such that $\forall \hat{x}, z \in \mathbb{R}^{n}, E \in \mathbb{R}^{m+p}, \forall t \geq 0$

$$
\alpha_{c, 1}(|\hat{x}|) \leq V_{c}(\hat{x}(t)) \leq \alpha_{c, 2}(|\hat{x}|)
$$

$\frac{\partial V_{c}(\hat{x})}{\partial \hat{x}} f_{o}(\hat{x}, \bar{\gamma}(\hat{x}), \bar{h}(\hat{x}+z)) \leq-\alpha_{c, 3}(|\hat{x}|)+\beta_{c}(|(z, E)|$

with $\alpha_{c, 1}, \alpha_{c, 2}, \alpha_{c, 3}, \beta_{c} \in \mathcal{K}$, and $\beta_{c}, \alpha_{c, 3}^{-1}$ Lipschitz;

$\left(A_{2}\right)$ There is an ISS Lyapunov function for $4 \mathrm{~b}$ such that $\forall z \in \mathbb{R}^{n}, E \in \mathbb{R}^{m+p}, \forall t \geq 0$

$$
\begin{aligned}
& \alpha_{o, 1}(|z|) \leq V_{o}(z(t)) \leq \alpha_{o, 2}(|z|) \\
& \frac{\partial V_{o}(z)}{\partial z} g\left(z, \theta_{1}\left(e_{u}\right), \theta_{2}\left(e_{y}\right), \hat{x}\right) \leq-\alpha_{o, 3}(|z|)+\beta_{o}(|E|)
\end{aligned}
$$

$$
\text { with } \alpha_{o, 1}, \alpha_{o, 2}, \alpha_{o, 3}, \beta_{o} \in \mathcal{K} \text {, and } \beta_{o}, \alpha_{o, 3}^{-1} \text { Lipschitz; }
$$

$\left(A_{3}\right) f_{o}, h$ and $\gamma$ are Lipschitz;

$\left(A_{4}\right) g$ is Lipschitz with respect to $\left(z, \theta_{1}\left(e_{u}\right), \theta_{2}\left(e_{y}\right)\right)$, uniformly in $\hat{x}$, and $\theta_{1}, \theta_{2}$ are Lipschitz.

Remark 1: $\left(A_{1}\right)$ ensures the asymptotic convergence to the origin of the observer, in absence of sampling errors and observation error, and an ISS property with respect to $z, e_{u}, e_{y} \cdot\left(A_{2}\right)$ ensures the asymptotic convergence to zero of the observation error in absence of sampling errors, and an ISS property with respect to $e_{u}, e_{y}$. Those two assumption suppose a separation principle between state estimation and control.

Since we are interested in the stabilisation of the observer state $\hat{x}$ and of the observation error state $z$, in the following we will assume that $X(0) \neq 0$.
Lemma 1: Under the Assumptions $\left(A_{1}\right),\left(A_{2}\right),\left(A_{3}\right),\left(A_{4}\right)$, the extended system $X=\left(\hat{x}^{T}, z^{T}\right)^{T}$ admits an ISS Lyapunov function $V(X)$ such that $\forall X \mathbb{R}^{2 n}, \forall E \in R^{n+p}$, $\forall t \geq 0$

$$
\begin{aligned}
a_{1}(|X|) & \leq V(X) \leq a_{2}(|X|) \\
\frac{\partial V(X)}{\partial X} G(X, E) & \leq-a_{3}(|X|)+b(|E|)
\end{aligned}
$$

with $a_{1}, a_{2}, a_{3}, b \in \mathcal{K}, b, a_{3}^{-1}, G$ Lipschitz.

Proof: Let us consider the candidate ISS Lyapunov function

$$
V(X)=\lambda_{c} V_{c}(\hat{x})+V_{o}(z)
$$

From $\left(A_{1}\right),\left(A_{2}\right)$,

$$
\begin{aligned}
a_{1}(|X|) & =\min _{|(\hat{x}, z)|=|X|} \lambda_{c} \alpha_{c, 1}(|\hat{x}|)+\alpha_{o, 1}(|z|) \\
& \leq \lambda_{c} \alpha_{c, 1}(|\hat{x}|)+\alpha_{o, 1}(|z|) \\
a_{2}(|X|) & =\max _{\left|\left(\hat{x}^{T}, z^{T}\right)\right|=|X|} \lambda_{c} \alpha_{c, 2}(|\hat{x}|)+\alpha_{o, 2}(|z|) \\
& \geq \lambda_{c} \alpha_{c, 2}(|\hat{x}|)+\alpha_{o, 2}(|z|)
\end{aligned}
$$

with $a_{1}, a_{2} \in \mathcal{K}$. Furthermore,

$$
\begin{aligned}
\frac{\partial V(X)}{\partial X} G(X, E)= & \left(\frac{\partial V(X)}{\partial \hat{x}} \frac{\partial V(X)}{\partial z}\right)\left(\begin{array}{c}
f_{o} \\
g
\end{array}\right) \\
\leq & \lambda_{c}\left(-\alpha_{c, 3}(|\hat{x}|)+\beta_{c}\left(\left|\left(z^{T}, E^{T}\right)^{T}\right|\right)\right) \\
& -\alpha_{o, 3}(|z|)+\beta_{o}\left(\left|E^{T}\right|\right) \\
\leq- & \left(\lambda_{c} \alpha_{c, 3}(|\hat{x}|)+\alpha_{o, 3}(|z|)-\lambda_{c} L_{\beta_{c}}|z|\right) \\
& +\lambda_{c} L_{\beta_{c}}|E|+\beta_{o}(|E|)
\end{aligned}
$$

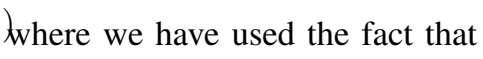

$$
\beta_{c}\left(\left|\left(z^{T}, E^{T}\right)^{T}\right|\right) \leq L_{\beta_{c}}\left|\left(z^{T}, E^{T}\right)^{T}\right| \leq L_{\beta_{c}}|E|+L_{\beta_{c}}|z| .
$$

It is always possible to choose $\lambda_{c}$ sufficiently small such that $\alpha_{o, 3}(|z|)-\lambda_{c} L_{\beta_{c}}|z|$ is a class $\mathcal{K}$ function with $z$ as variable. Since we are considering 1 -norm

$$
a_{3}(|X|)=\min _{|X|}\left\{\lambda_{c} \alpha_{c, 3}(|X|), \alpha_{o, 3}(X)-\lambda_{c} L_{\beta_{c}}|X|\right\} .
$$

To show that $a_{3}^{-1}$ is Lipschitz, note first that since $\alpha_{o, 3}^{-1}$ is Lipschitz

$$
\alpha_{o, 3}(|z|) \geq \frac{1}{L_{\alpha_{o, 3}^{-1}}}|z| .
$$

Moreover, one can compute an upper bound on the derivative of $\left(\alpha_{o, 3}(|\cdot|)-\lambda_{c} L_{\beta_{c}}|\cdot|\right)^{-1}$, since

$$
\frac{d}{d|z|}\left(\alpha_{o, 3}(|z|)-\lambda_{c} L_{\beta_{c}}|z|\right)^{-1} \leq \frac{L_{\alpha_{o, 3}^{-1}}}{1-\lambda_{c} L_{\alpha_{o, 3}^{-1}} L_{\beta_{c}}}
$$

Hence it is always possible to choose $\lambda_{c}$ sufficiently small such that $\left(\alpha_{o, 3}(|\cdot|)-\lambda_{c} L_{\beta_{c}}|\cdot|\right)^{-1}$ is a class $\mathcal{K}$ function with Lipschitz constant

$$
L_{a_{3}^{-1}}=\max \left\{\frac{\lambda_{c}}{L_{\alpha_{c, 3}}}, \frac{L_{\alpha_{o, 3}^{-1}}}{1-\lambda_{c} L_{\alpha_{o, 3}^{-1}} L_{\beta_{c}}}\right\} .
$$


Furthermore,

$$
b(|E|)=L_{\beta_{c}}|E|+\beta_{o}(|E|)
$$

which is Lipschitz with constant $L_{b}=L_{\beta_{c}}+L_{\beta_{o}}$. Finally, thanks to $\left(A_{3}\right),\left(A_{4}\right), G(X, E)$ is Lipschitz.

In the following section we are interested in providing sufficient conditions on the stabilisation of a nonlinear system using the event trigger paradigm. The key concept will be the ISS of both the closed-loop system and of the observer dynamics. For, we introduce the following lemmas.

Lemma 2: If the observer and the error dynamics verify $\left(A_{1}\right),\left(A_{2}\right),\left(A_{3}\right),\left(A_{4}\right)$, then there exist a $\sigma>0$ such that any sampling policy ensuring $|E| \leq \sigma|X|$, leads to asymptotic convergence of the overall system to the origin.

Proof: From Lemma 1, the existence of an ISS Lyapunov function $V$ is ensured. Since $a_{3}^{-1}$ and $b$ are Lipschitz, $a_{3}(|X|) \geq \frac{1}{L_{a_{3}^{-1}}}|X|$ and
$\quad-a_{3}(|X|)+b(\sigma|X|) \leq-\left(\frac{1}{L_{a_{3}^{-1}}}-L_{b} \sigma\right)|X|$.

Therefore for all $\sigma \in\left(0, \frac{1}{L_{a_{3}^{-1}} L_{b}}\right)$ the system (4) converge asymptotically to the origin.

Remark 2: Under the hypothesis that $a_{2}, a_{1}^{-1}$ are Lipschitz, one can prove exponential convergence of (4). In fact, since

$$
\frac{1}{L_{a_{1}^{-1}}}|X|<a_{1}(|X|)<V(|X|)
$$

one has that

$$
\dot{V}(|X|) \leq-\left(\frac{1}{L_{a_{3}^{-1}}}-L_{b} \sigma\right)|X| .
$$

Therefore

$$
\dot{V}(|X|) \leq-\left(\frac{1}{L_{a_{3}^{-1}}}-L_{b} \sigma\right) L_{a_{1}^{-1}} V(|X|) .
$$

Remark 3: The choice $\sigma \in\left(0, \frac{1}{L_{a_{3}^{-1}} L_{b}}\right)$ represents a trade-off between the sampling rate and the convergence rate.

Since $|E|<\sigma|X|$, using the norm equivalence there exists a $\sigma^{\prime}>0$ such that $\|E\|<\sigma^{\prime}\|X\|$ implies $|E|<\sigma|X|$.

Lemma 3: For every $\kappa_{i}>0$ there is a minimal time $\tau_{\min }>0$ such that if $|E| \leq \sigma|X|$, then $\forall t_{k}, \forall t \in$ $\left[t_{k}, t_{k}+\tau_{\min }\right)$ the following inequalities are verified

$$
\begin{aligned}
\left\|\gamma_{i}(\hat{x}(t))-\gamma_{i}\left(\hat{x}\left(t_{k}\right)\right)\right\| & \leq \kappa_{i}\|X\| \\
\| h_{j}(\hat{x}(t)+z(t))-h_{j}\left(\hat{x}\left(t_{k}\right)+z\left(t_{k}\right) \|\right. & \leq \kappa_{j}\|X\| .
\end{aligned}
$$

Proof: In the following we assume $X \neq 0$. The argument follows the proof of Theorem 1 in [12]. Denoting $e_{u_{i}}=\gamma_{i}(\hat{x}(t))-\gamma_{i}\left(\hat{x}\left(t_{k}\right)\right)$, one works out

$$
\begin{aligned}
\frac{d}{d t} \frac{\left\|e_{u_{i}}\right\|}{\|X\|} & =\frac{e_{u_{i}}^{T} \dot{e}_{u_{i}}}{\left\|e_{u_{i}}\right\|\|X\|}-\frac{X^{T} \dot{X}\left\|e_{i}\right\|}{\|X\|^{3}} \\
& \leq \frac{\left\|e_{u_{i}}\right\|\left\|\dot{e}_{u_{i}}\right\|}{\left\|e_{u_{i}}\right\|\|X\|}+\frac{\|\dot{X}\|\left\|e_{i}\right\|}{\|X\|^{2}} .
\end{aligned}
$$

Since $\left\|\dot{e}_{u_{i}}\right\| \leq L_{\gamma_{i}}\|\dot{\hat{x}}\| \leq L_{\gamma_{i}}\|\dot{X}\|$,

$$
\frac{d}{d t} \frac{\left\|e_{u_{i}}\right\|}{\|X\|} \leq \frac{\|\dot{X}\|}{\|X\|}\left(L_{\gamma_{i}}+\frac{\left\|e_{i}\right\|}{\|X\|}\right)
$$

Moreover, $G$ is Lipschitz, so that

$$
\frac{d}{d t} \frac{\left\|e_{u_{i}}\right\|}{\|X\|} \leq \frac{L_{G}(\|X\|+\|E\|)}{\|X\|}\left(L_{\gamma_{i}}+\frac{\left\|e_{i}\right\|}{\|X\|}\right)
$$

Since $\|E\|<\sigma^{\prime}\|X\|$,

$$
\frac{d}{d t} \frac{\left\|e_{u_{i}}\right\|}{\|X\|} \leq L_{G}\left(1+\sigma^{\prime}\right)\left(L_{\gamma_{i}}+\frac{\left\|e_{i}\right\|}{\|X\|}\right) .
$$

At each reset time one has $e_{u_{i}}=0$. Using the comparison lemma with the differential equation

$$
\dot{y}=L_{G}\left(1+\sigma^{\prime}\right)\left(L_{\gamma_{i}}+y\right), \quad y(0)=0
$$

one has

$$
\frac{\left\|e_{u_{i}}(t)\right\|}{\|X(t)\|} \leq\left(e^{L_{G}\left(1+\sigma^{\prime}\left(t-t_{k}\right)\right.}-1\right) L_{\gamma_{i}} .
$$

Therefore the inequality

$$
\left\|\gamma_{i}(\hat{x}(t))-\gamma_{i}\left(\hat{x}\left(t_{k}\right)\right)\right\|>\kappa_{i}\|X\|
$$

can not be true before time

$$
\tau_{\min }^{i}=\frac{1}{L_{G}\left(1+\sigma^{\prime}\right)} \ln \left(1+\frac{\kappa_{i}}{L_{\gamma_{i}}}\right) .
$$

Analogously, for

$$
\| h_{j}(\hat{x}(t)+z(t))-h_{j}\left(\hat{x}\left(t_{k}\right)+z\left(t_{k}\right)\left\|>\kappa_{j}\right\| X \|\right.
$$

gives for the sensors

$$
\tau_{\min }^{j}=\frac{1}{L_{G}\left(1+\sigma^{\prime}\right)} \ln \left(1+\frac{\kappa_{j}}{L_{h_{i}}}\right) .
$$

Let us define the triggering function at each node

$$
\begin{aligned}
& t_{k+1}^{i}=\min _{t}\left\{t \geq t_{k}^{i}+\tau_{\min }^{i}, \mid\left\|u_{i}(t)-u_{i}\left(t_{k}^{i}\right)\right\|>\kappa_{i}\|X\|\right\} \\
& t_{k+1}^{j}=\min _{t}\left\{t \geq t_{k}^{j}+\tau_{\min }^{j}, \mid\left\|y_{j}(t)-y_{j}\left(t_{k}^{i}\right)\right\|>\kappa_{j}\|X\|\right\} .
\end{aligned}
$$

Remark 4: From Lemma 3, $t_{k+1}^{i}=\min _{t}\left\{t \geq t_{k}^{i}+\tau_{\min }^{i}, \mid\right.$ $\left.\left\|u_{i}(t)-u_{i}\left(t_{k}^{i}\right)\right\|>\kappa_{i}\|X\|\right\}=t_{k+1}^{i}=\min _{t}\left\{t \geq t_{k}^{i}, \mid\right.$ $\left.\left\|u_{i}(t)-u_{i}\left(t_{k}^{i}\right)\right\|>\kappa_{i}\|X\|\right\}$.

Lemma 4: If $\sum_{\{1, \cdots, r\} \cup\{1, \cdots, q\}} \kappa_{i} \leq \sigma^{\prime}$ then (6) and (7) ensure $\|E\| \leq \sigma\|X\|$. 
Proof: from (6) and (7)

$$
\|E\| \leq \sum_{\{1, \cdots, r\} \cup\{1, \cdots, q\}} \kappa_{i}\|X\| \leq \sigma^{\prime}\|X\| .
$$

The proposed triggering conditions allow asymptotic convergence with a nonzero minimum inter-event time. Unfortunately, they are not implementable on a network for two reasons. The first is that $X$ is not available, since the observation error is not known. The second is that sensors do not communicate among them nor receive information from the observer-based controller. Nevertheless, considering the following modified triggering conditions

$t_{k+1}^{i}=\min _{t}\left\{t \geq t_{k}^{i}+\tau_{\min }^{i}, \mid\left\|u_{i}(t)-u_{i}\left(t_{k}^{i}\right)\right\|>\frac{\kappa_{i}}{L_{\gamma_{i}}}\left\|\gamma_{i}(\hat{x})\right\|\right\}$

$t_{k+1}^{j}=\min _{t}\left\{t \geq t_{k}^{j}+\tau_{\min }^{j}, \mid\left\|y_{j}(t)-y_{j}\left(t_{k}^{i}\right)\right\|>\frac{\kappa_{j}}{2 L_{h}}\left\|y_{j}\right\|\right\}$

this approach can be used on a network, allowing asymptotic convergence and a nonzero minimal inter-event time, using only information available at each node, as stated by the main contribution of this work.

Theorem 1: If $\left(A_{1}\right),\left(A_{2}\right),\left(A_{3}\right),\left(A_{4}\right)$ are verified, and the sampling instants are defined by (8), (9), then the origin of the closed-loop system (4) is asymptotically stable and there exists a nonzero minimum inter-event time for each node.

Proof: Under the hypotheses of the theorem, Lemma 1 applies. Since $\frac{\left\|y_{j}\right\|}{2 L_{h}}<\|X\|$ and $\frac{\left\|u_{i}\right\|}{L_{\gamma_{i}}}<\|X\|$ and $\forall i \in$ $\{1, \cdots, r\}$ from Lemma 3 one can state that between $t_{k_{i}}$ and $t_{k_{i}}+\tau_{\text {min }}^{i},\left\|u_{i}(t)-u_{i}\left(t_{k}^{i}\right)\right\|>\kappa_{i}\|X\|$, while $\forall j \in\{1, \cdots, q\}$ between $t_{k_{j}}$ and $t_{k_{j}}+\tau_{\min }^{j}$, one has $\left\|y_{j}(t)-y_{j}\left(t_{k_{j}}\right)\right\|>$ $\kappa_{j}\|X\|$.

Therefore, $\|E\|<\sigma^{\prime}\|X\|$. Using Lemma 2, there is asymptotic convergence of (4) to the origin.

\section{EXAMPLES OF Systems FitTing INTO THE PROPOSED FRAMEWORK}

\section{A. Linear Systems}

Let us consider a detectable and stabilizable linear system

$$
\begin{aligned}
\dot{x} & =A x+B u \\
y & =x
\end{aligned}
$$

with

$$
\dot{\hat{x}}=A \hat{x}+B u+L C(\bar{x}-\hat{x})
$$

a Luenberger observer. With control $K \overline{\hat{x}}$, one gets

$$
\begin{aligned}
\dot{\hat{x}} & =(A+B K) \hat{x}+B K(\overline{\hat{x}}-\hat{x})+L C z-L C(x-\bar{x}) \\
\dot{z} & =(A-L C) z+L C(x-\bar{x}) .
\end{aligned}
$$

Since $A+B K$ and $A-L C$ are Hurwitz, it is possible to find an ISS Lyapunov function for the extended system.

\section{B. Nonlinear Lipschitz Systems}

Let us consider a nonlinear Lipschitz system

$$
\begin{aligned}
& \dot{x}=A x+B u+\phi(x, u) \\
& y=C x .
\end{aligned}
$$

Several results are available for the observer synthesis of nonlinear Lipschitz systems when the control and the output are implemented in a continuous fashion. We consider an observer of the form

$$
\dot{\hat{x}}=A x+B K \hat{x}+\phi(x, K \hat{x}) .
$$

Hence, the extended closed-loop system is

$$
\begin{aligned}
\dot{\hat{x}} & =A+\hat{x}+B K \hat{x}+\phi(\hat{x}, K \hat{x})+L C z \\
\dot{z} & =(A-L C) z+\phi(x, K \hat{x})-\phi(\hat{x}, K \hat{x}) .
\end{aligned}
$$

To implement an event-triggered control strategy, we need to consider the following structural properties.

$\left(H_{1}\right)\left\|\phi\left(x_{1}, u\right)-\phi\left(x_{2}, u\right)\right\| \leq \rho\left\|x_{1}-x_{2}\right\|, \forall u \in \mathbb{R}^{p}$, $\left(x_{1}, x_{2}\right) \in \mathbb{R}^{2 n}$

$\left(H_{2}\right) \quad\|\phi(x, u)\| \leq \rho\|x\|, \forall u \in \mathbb{R}^{p}$;

$\left(H_{3}\right)$ There exist a gain $K$ such that $u=K x$ for the system (13) and there exist a quadratic Lyapunov function

$$
V_{c}(x)=x^{T} P_{c} x, \quad \dot{V}_{c}(x) \leq-\eta_{c} x^{T} x
$$

with $P_{c}=P_{c}^{T}>0, \eta_{c}>0$;

$\left(H_{4}\right)$ There exist gain $L$ such that for $(14 \mathrm{~b})$ and there exist quadratic Lyapunov function for the $z$ dynamic

$$
V_{o}(z)=z^{T} P_{o} z, \quad \dot{V}_{o}(z) \leq-\eta_{o} z^{T} z
$$

with $P_{o}=P_{o}^{T}>0, \eta_{o}>0$.

In $\left(H_{2}\right)$, for $\rho=0$ we have a linear system, and the existence of $V_{c}, V_{o}$ derive from the stabilizability and the detectability. Moreover, there always exists a $\rho_{\max }>0$ small enough such that the proposed Lyapunov function exist forall $\rho \in\left[0, \rho_{\max }\right]$. For other (more complex) conditions of existence of $V_{c}, V_{o}$ verifying [15], [16, see for instance [10].

Lemma 5: If $\left(H_{1}\right),\left(H_{2}\right),\left(H_{3}\right),\left(H_{4}\right)$ are verified, then the proposed observer and the observation error verify $\left(A_{1}\right),\left(A_{2}\right),\left(A_{3}\right),\left(A_{4}\right)$.

Proof: When subject to the trigger conditions, the observer has the following dynamics

$$
\dot{\hat{x}}=(A+B K) \hat{x}+B K(\hat{x}-\overline{\hat{x}})+L C z+L C(\bar{z}-z) .
$$

Let us consider the candidate ISS Lyapunov function

$$
2 \sqrt{\lambda_{\min }\left(P_{c}\right)}\|\hat{x}\| \leq 2 \sqrt{V_{c}(\hat{x})} \leq 2 \sqrt{\lambda_{\max }\left(P_{c}\right)}\|\hat{x}\|
$$

having derivative

$$
\begin{aligned}
& \frac{d}{d t} 2 \sqrt{\hat{x}^{T} P_{c} \hat{x}}=\frac{1}{\sqrt{\hat{x}^{T} P_{c} \hat{x}}}\left(-\hat{x}^{T} Q \hat{x}+2 \hat{x}^{T} P \phi(\hat{x}, u)\right) \\
& +\frac{1}{\sqrt{\hat{x}^{T} P_{c} \hat{x}}}\left(2 \hat{x}^{T} P(B K(x-\bar{x})+L C z-L C(z-\bar{z}))\right.
\end{aligned}
$$


where $Q=-(A+B K)^{T} P+P(A+B K)$. In virtue of $\left(H_{1}\right)$, one can write

$$
\begin{aligned}
& \frac{d}{d t} 2 \sqrt{\hat{x}^{T} P_{c} \hat{x}} \leq-\frac{\eta_{c}\|\hat{x}\|^{2}}{\sqrt{\hat{x}^{T} P_{c} \hat{x}}} \\
& +\frac{1}{\sqrt{\hat{x}^{T} P_{c} \hat{x}}}[\|P\|\|\hat{x}\|(\|B K\|\|(x-\bar{x})\| \\
& \quad+\|L C\|\|z\|+\|L C\|\|(z-\bar{z})\|)] \\
& \leq \frac{-\eta_{c}}{\sqrt{\lambda_{\max }\left(P_{c}\right)}}\|\hat{x}\| \\
& +\frac{(\|B K\|\|(x-\bar{x})\|+\|L C\|\|z\|+\|L C\|\|(z-\bar{z})\|)}{\sqrt{\lambda_{\min }\left(P_{c}\right)}}
\end{aligned}
$$

which verifies assumption $\left(A_{1}\right)$. Analogously, using the candidate ISS Lyapunov function $2 \sqrt{V_{o}}$, one can prove that $\left(A_{2}\right)$ holds. Furthermore, it is trivial to show that $\left(H_{1}\right),\left(H_{2}\right)$ imply $\left(A_{3}\right),\left(A_{4}\right)$.

Therefore, applying Lemma 1 to the system (12), and using Theorem 11 to the event-triggered observer-based controller ensures asymptotic convergence to the origin.

Corollary 1: If $\left(H_{1}\right),\left(H_{2}\right),\left(H_{3}\right),\left(H_{4}\right)$ are verified, the event-triggered control policy (8), (9) and the control $u=$ $K \overline{\hat{x}}$ ensure the asymptotic stability of the closed-loop system 14.

Proof: Lemma 5 ensures that $\left(A_{1}\right),\left(A_{2}\right),\left(A_{3}\right),\left(A_{4}\right)$ are verified. Then one applies Theorem 1 to the system 14 .

\section{Simulations}

The proposed methodology will be applied to a robot with a flexible link, used as a benchmark example in several papers dealing with Lipschitz observers (see for instance [11], [1], [10]). The dynamics are in the form (12), with

$$
\begin{aligned}
& \dot{x}=A x+\phi(x, u)+B K \overline{\hat{x}} \\
& \dot{\hat{x}}=A \hat{x}+\phi(\hat{x}, u)+B K \overline{\hat{x}}+L C \bar{z} \\
& y=C x
\end{aligned}
$$

where

$$
\begin{aligned}
& A=\left(\begin{array}{cccc}
0 & 1 & 0 & 0 \\
-48.6 & -1.25 & 48.6 & 0 \\
0 & 0 & 0 & 1 \\
19.5 & 0 & -19.5 & 0
\end{array}\right), B=\left(\begin{array}{llll}
0 & 21.6 & 0 & 0
\end{array}\right)^{T} \\
& C=\left(\begin{array}{llll}
1 & 0 & 0 & 0 \\
0 & 1 & 0 & 0
\end{array}\right), \quad \phi=\left(\begin{array}{lllll}
0 & 0 & 0 & 3.3 \sin x_{3}
\end{array}\right)^{T} .
\end{aligned}
$$

One considers the control $u=K \overline{\hat{x}}$, with

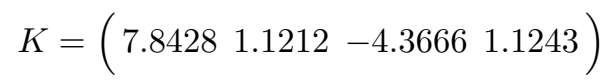

and the observer (13), with

$$
L=\left(\begin{array}{cc}
9.3334 & 1.0001 \\
-48.7804 & 22.3665 \\
-0.0524 & 3.3194 \\
19.4066 & -0.3167
\end{array}\right) \text {. }
$$

The closed-loop equations are in the form (14). The simulations have been performed considering the initial states

$$
x(0)=\left(\begin{array}{llll}
1 & 1 & 1 & 1
\end{array}\right)^{T}, \quad \hat{x}(0)=\left(\begin{array}{llll}
0 & 0 & 0 & 0
\end{array}\right)^{T} .
$$

The theoretical values obtained on the triggering policy can be used but are too restrictive, due to the over-approximation on the convergence rate of the nonlinear observer and on the triggering parameter estimations. Via simulations it is possible to better tune the triggering parameters. It is worth noting that there is an order of magnitude of 100 between the theoretical value and the practical ones. We compared the result of a system controlled using triggering policy

$$
\begin{aligned}
& t_{k_{i}+1}=\min _{t}\left\{t \geq t_{k_{i}}+0.01,|| u_{i}(t)-u_{i}\left(t_{k_{i}}\right)|>0.2| u_{i}(t) \mid\right\} \\
& t_{k_{j}+1}=\min _{t}\left\{t \geq t_{k_{j}}+0.01,|| y_{j}(t)-y_{j}\left(t_{k_{j}}\right)|>0.2| y_{j}(t) \mid\right\}
\end{aligned}
$$

with the case in which $t_{k_{i}+1}=t_{k_{i}}+0.05$. The simulations show that for $t \in[0,2] \mathrm{s}$ the system and observer are closed to the equilibrium, while at $t=2 \mathrm{~s}$ an impulse drives the system away from equilibrium. Then, for $t \in[2,15] \mathrm{s}$, the system is stabilized at the origin by the proposed observerbased controller.

Figs. 2, 3 show the convergence of the observer and the stabilization at the origin of the overall system. We can note that the event triggering is relatively slower with respect to the periodic sampling, but introduces a lower peaking. When confronting the number of triggers in Fig.s $4 \mathrm{a}, 4 \mathrm{~b}$, it is clear that the number of communications is greater when considering the periodic sampling, so justifying the interest of the proposed event-triggering scheme. It is worth noting that the advantage of the method appears more clearly for output communications. As already noted, this is due to the fact that the observation and the control communications are done only when necessary. The comparison of Figs. $4 \mathrm{a}, 4 \mathrm{~b}$ illustrates a trade-off between "intelligence" in the sensor and actuator, and the communication burden.

\section{CONCLUSION}

In this paper we have presented an event-triggered observer-based controller for a class of nonlinear systems. Sufficient conditions in term of ISS stability for the observer and the observation error dynamics are given for designing an event-triggering mechanism ensuring the asymptotic convergence to the origin of the closed-loop system state. A particular subclass is that of systems with Lipschitz nonlinearities. The relevance of the approach has been highlighted by simulations of a robot with a flexible link, where the triggering parameters have been appropriately tuned.

Further work will include a practical way of determining theoretically a good choice of triggering parameters. Furthermore, even thought the hypotheses on the state and on the observer imply a separation principle (convergence of the observer without assumption on the trajectory of the state) when considering a continuous feedback, this property is lost when introducing the triggering policy. Since this is not the case when considering periodic sampling, an interesting 
a)

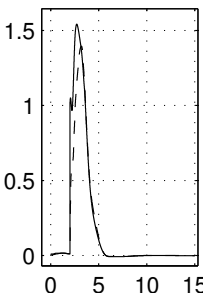

e)
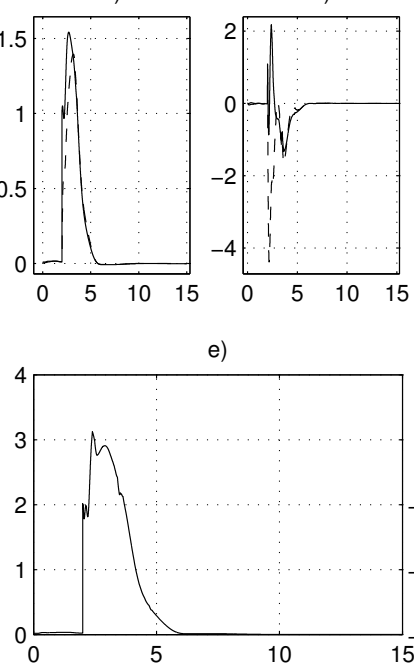

c)
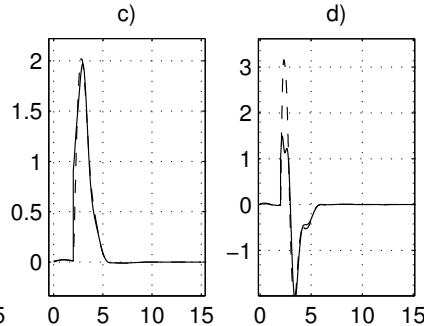

f)

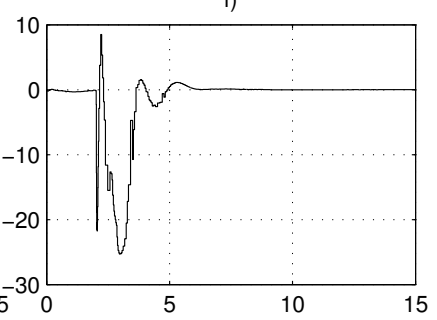

Fig. 2. System and observer state with the event-triggering: a) $x_{1}, \hat{x}_{1}$; b) $x_{2}, \hat{x}_{2}$; c) $x_{3}, \hat{x}_{3}$; d) $x_{4}, \hat{x}_{4}$; e) $\|x\|$; f) $u$.
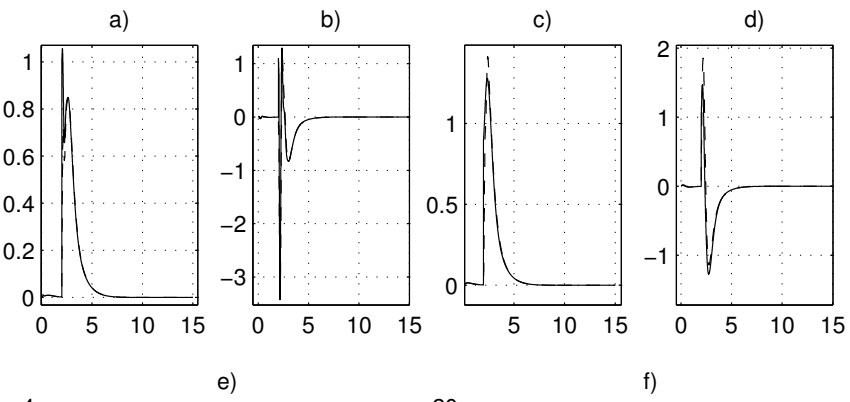

e)

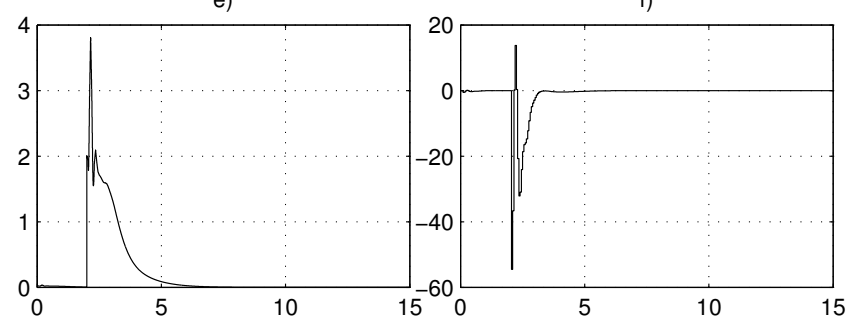

Fig. 3. System and observer state with periodic sampling: a) $x_{1}, \hat{x}_{1}$; b) $x_{2}, \hat{x}_{2}$; c) $x_{3}, \hat{x}_{3}$; d) $x_{4}, \hat{x}_{4}$; e) $\|x\|$; f) $u$.
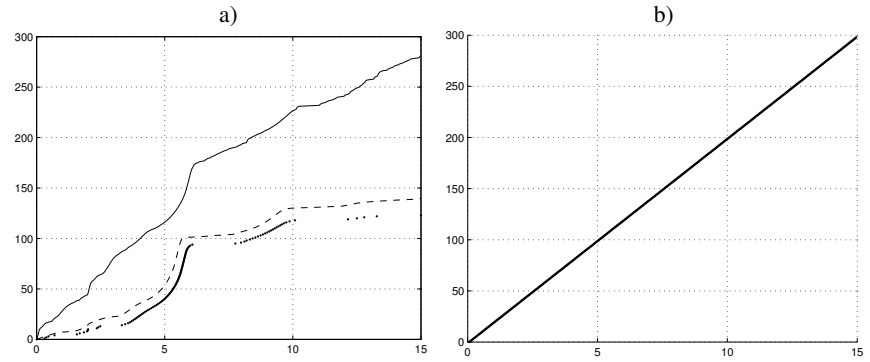

Fig. 4. Number of triggers with the a) Proposed event triggered policy; b) Periodic sampling. $u_{1}$ (solid), $y_{1}$ (dashed), $y_{2}$ (dotted).

question to address is: Can we ensure a separation principle when using event-triggered control policies?

\section{REFERENCES}

[1] C. Aboky, G. Sallet, and J. C. Vivalda, Observers for Lipschitz NonLinear Systems, International Journal of Control, Vol. 75, No. 3, pp. 204-212, 2002.
[2] K.J. Åstrom, and B. Wittenmark, Computer Controlled Systems, Prentice Hall, 1997.

[3] K.J. Åstrom, and B. Bernhardsson, Systems with Lebesgue sampling, Directions in Mathematical Systems Theory and Optimization, pp. 113, Springer Berlin, Heidelberg, 2003.

[4] M.C.F. Donkers, and W.P.M.H. Heemels, Output-Based EventTriggered Control with Guaranteed-Gain and Improved and Decentralized Event-Triggering, IEEE Transactions on Automatic Control, Vol. 57, No. 6, pp. 1362-1376, 2012.

[5] W.P.M.H. Heemels, J.H. Sandee, and P.P.J. Van Den Bosch, Analysis of Event-Driven Controllers for Linear Systems, International Journal of Control, Vol. 81, No. 4, pp. 571-590, 2008.

[6] W.P.M.H. Heemels, K.H. Johansson, and P. Tabuada, An Introduction to Event-Triggered and Self-Triggered Control. Proceedings of the $51^{\text {st }}$ Conference on Decision and Control, pp. 3270-3285), 2012.

[7] K. H. Johansson, M. Egerstedt, J. Lygeros and S. Sastry, On the Regularization of Zeno Hybrid Automata, Systems \& Control Letters, Vol. 38, No. 3, pp. 141-150, 1999.

[8] D. Lehmann, and J. Lunze, Event-Based Output-Feedback Control, Proceedings of the $19^{\text {th }}$ Mediterranean Conference on Control and Automation, pp. 982-987, 2011.

[9] J. Lunze, and D. Lehmann, A State-Feedback Approach to EventBased Control, Automatica, Vol. 46, No. 1, pp. 211-215, 2010.

[10] P. R. Pagilla, and Y. Zhu, Controller and Observer Design for Lipschitz Nonlinear Systems, Proceedings of the 2004 American Control Conference, pp. 2379-2384, 2004.

[11] I. R. Raghavan \& J. K. Hedrick, Observer Design for a Class of Nonlinear Systems, International Journal of Control, Vol. 1, pp. 171185, 1994.

[12] P. Tabuada, Event-Triggered Real-Time Scheduling of Stabilizing Control Tasks, IEEE Transactions on Automatic Control, Vol. 52, No. 9, pp. 1680-1685, 2007.

[13] P. Tallapragada, and N. Chopra, Event-Triggered Dynamic Output Feedback Control of LTI Systems over Sensor-Controller-Actuator Networks, Proceedings of the $52^{\text {nd }}$ Conference on Decision and Control, pp 4625-4630, 2013.

[14] X. Wang, and M.D. Lemmon, Event-Triggering in Distributed Networked Control Systems, IEEE Transactions on Automatic Control, Vol. 56, No. 3, pp. 586-601, 2011. 